\title{
Trends in epidemiologic characteristics of end-stage renal disease from 2019 Korean Renal Data System (KORDS)
}

\author{
Yu Ah Hong ${ }^{1}$, Tae Hyun Ban ${ }^{1}$, Chae-Yeong Kang ${ }^{2}$, Sun Deuk Hwang ${ }^{3}$, Sun Ryoung Choi ${ }^{4}$, Hajeong Lee ${ }^{5}$, \\ Hee-Yeon Jung ${ }^{6}$, Kyeongmin Kim ${ }^{7}$, Young Eun Kwon ${ }^{8}$, Su Hyun $\mathrm{Kim}^{9}$, Tae Hee Kim ${ }^{10}$, Ho-Seok Koo ${ }^{10}$, \\ Chang-Yun Yoon ${ }^{11}$, Kiwon $\mathrm{Kim}^{12}$, Jongha Park ${ }^{13}$, Yong Kyun Kim ${ }^{1}$ \\ ${ }^{1}$ Department of Internal Medicine, College of Medicine, The Catholic University of Korea, Seoul, Republic of Korea \\ ${ }^{2}$ The Korean Society of Nephrology, Seoul, Republic of Korea \\ ${ }^{3}$ Department of Internal Medicine, Inha University College of Medicine, Incheon, Republic of Korea \\ ${ }^{4}$ Department of Internal Medicine, Hallym University Dongtan Sacred Heart Hospital, Hwaseong, Republic of Korea \\ ${ }^{5}$ Department of Internal Medicine, Seoul National University Hospital, Seoul, Republic of Korea \\ ${ }^{6}$ Department of Internal Medicine, School of Medicine, Kyungpook National University, Kyungpook National University Hospital, Daegu, \\ Republic of Korea \\ ${ }^{7}$ Department of Internal Medicine, Daejeon Eulji Medical Center, Eulji University, Daejeon, Republic of Korea \\ ${ }^{8}$ Department of Internal Medicine, Myongji Hospital, Hanyang University College of Medicine, Goyang, Republic of Korea \\ ${ }^{9}$ Department of Internal Medicine, Chung-Ang University Hospital, Seoul, Republic of Korea \\ ${ }^{10}$ Department of Internal Medicine, Inje University College of Medicine, Busan, Republic of Korea \\ ${ }^{11}$ Yoon's Medical Clinic Dialysis Center, Seoul, Republic of Korea \\ ${ }^{12}$ Seoul One Clinic, Anyang, Republic of Korea \\ ${ }^{13}$ Department of Internal Medicine, Ulsan University Hospital, University of Ulsan College of Medicine, Ulsan, Republic of Korea
}

Background: The Korean Society of Nephrology (KSN) has maintained a nationwide end-stage renal disease (ESRD) registry data from Korean Renal Data System (KORDS) since 1985, as the representative registry of ESRD patients in Korea. This review is aimed to update the status of domestic ESRD and to provide evidence on the direction of dialysis therapy.

Methods: The KORDS Committee of KSN has collected data on dialysis centers and patients through an online registry program, and the data from 1986 to 2019 were analyzed.

Results: The incidence and prevalence of ESRD patients in Korea are increasing. The ESRD population numbered more than 100,000 in 2019 , doubling during the 10 years since 2010 . The proportion of diabetes mellitus as a major cause of ESRD seems to

Received: October 27, 2020; Revised: November 20, 2020; Accepted: November 25, 2020

Editor: Tae-Hyun Yoo, Yonsei University, Seoul, Republic of Korea

Correspondence: Jongha Park

Division of Nephrology, Department of Internal Medicine, Ulsan University Hospital, University of Ulsan College of Medicine, 873

Bangeojinsunhwando-ro, Dong-gu, Ulsan 44033, Republic of Korea. E-mail: jonghaparkmd@gmail.com

ORCID: https://orcid.org/0000-0002-1461-9483

Yong Kyun Kim

Division of Nephrology, Department of Internal Medicine, St. Vincent's Hospital, College of Medicine, The Catholic University of Korea, 93 Jungbudaero, Paldal-gu, Suwon 16247, Republic of Korea. E-mail: drkimyk@catholic.ac.kr

ORCID: https://orcid.org/0000-0002-1871-3549

Yu Ah Hong and Tae Hyun Ban contributed equally to this work as co-first authors.

Copyright (@ 2021 by The Korean Society of Nephrology

() This is an Open Access article distributed under the terms of the Creative Commons Attribution Non-Commercial and No Derivatives License (http:// creativecommons.org/licenses/by-nc-nd/4.0/) which permits unrestricted non-commercial use, distribution of the material without any modifications, and reproduction in any medium, provided the original works properly cited. 
have reached a plateau. The increasing number of elderly dialysis patients is a constant trend, with more than half for the proportion of patients older than 65 years old in 2019. All-cause mortality decreased for the last approximately 20 years, regardless of sex, age, and cause of ESRD. The 5-year patient survival rate in both hemodialysis and peritoneal dialysis increased from 2001 to 2013 . Since 2013, the patient survival rates in peritoneal dialysis were similar to those in hemodialysis. Cardiovascular complications were the leading cause of death in ESRD patients.

Conclusion: The incidence and prevalence of Korean ESRD patients have increased over time, although patient survival has also steadily increased. The establishment of a surveillance method to address the major cause of mortality in ESRD patients will help improve outcomes.

Keywords: Chronic kidney failure, Incidence, Mortality, Prevalence

\section{Introduction}

The incidence and prevalence of end-stage renal disease (ESRD) requiring renal replacement therapy (RRT) either by dialysis or kidney transplantation (KT) have progressively increased in countries around the world, including Korea $[1,2]$. Identifying ESRD incidence and prevalence is of vast importance so that policymakers and health care providers can develop public health care plans for ESRD patients. The Korean Society of Nephrology (KSN) launched a nationwide ESRD patient registry, Korean Renal Data System (KORDS), in 1985, which has continued for over 35 years. The goals of the KORDS are as follows: (1) to estimate the numbers and distributions of ESRD patients; (2) to determine the characteristics of ESRD and dialysis therapy and their complications or outcomes based on scientific evidence; and (3) to improve the quality of dialysis therapy and support public health decisions related to ESRD [3]. Every year, the KSN releases an annual report from KORDS, which presents an overview of the incidence and prevalence of RRT including KT, the characteristics of patients, and the survival of patients on RRT in Korea. In this article, we provide a summary of the 2019 KORDS annual report. This article presents the most recent data on the epidemiology of RRT for ESRD, which focuses on the incidence and prevalence of RRT and ESRD mortality trends in Korea. More detailed data from the KORDS are available through the KSN website (http://www.ksn.or.kr).

\section{Methods}

The KORDS was populated with responses from mailed paper questionnaires from 1985 to 1994. An electronic questionnaire with dial-up modem file transfer or diskette mailing was used from 1995 to 2000 [4]. An internetbased questionnaire for dialysis patient registry has been administered by the KSN since 2001 via an online registry program on the KSN website and is updated yearly [5]. Enrollment in the registry is voluntarily updated by members of the KSN. The registry program has collected data throughout the years, and the collected data include dialysis center information, dates of hemodialysis (HD) or peritoneal dialysis (PD) initiation, newly developed comorbid diseases, vascular access, dialysis doses, medications including erythropoiesisstimulating agents and phosphorus-controlling agents, laboratory data, dialysis adequacy, rehabilitation status, and outcomes for each patient on HD, on PD, or who had undergone KT. Among these data, this study analyzed the changes in incidence and prevalence rates and outcomes in ESRD patients from 1986 to 2019. Because the survival data from 1986 to 2000 were insufficient, the analyses of incidence and prevalence of ESRD patients were performed from 1986 to 2019, but the analyses of outcomes were performed from 2001 to 2019. The KT recipients were excluded from outcome analysis. A flow diagram for patient selection is presented in Supplementary Fig. 1 (available online). Trends in mortality rates are presented for the patients treated each year according to the number of patient-years at risk. Unadjusted survival rates were calculated using the KaplanMeier method, and absolute mortality rates were presented per 1,000 person-years of follow-up. All statistical analyses of survival data were analyzed using SAS (version 9.4; SAS Institute, Cary, NC, USA) and R (version 4.0.1; R Foundation for Statistical Computing, Vienna, Austria). 


\section{Results}

\section{Incidence and prevalence of RRT in adults in Korea}

At the end of 2019 in Korea, the total number of new patients who started RRT for ESRD was 18,642, comprising 15,587 (83.6\%) with HD, 762 (4.1\%) with PD, and 2,293 (12.3\%) with KT (Fig. 1A). The total number of patients starting RRT was more than 10,000 in 2011, illustrating the continuously increasing trend. In particular, the proportion of ESRD patients who selected HD as the initial treatment modality was steadily growing. The percentage of incident HD patients increased from less than $70 \%$ before 2008 to more than $80 \%$ after 2014 and was maintained at around 85\% in 2019 (Fig. 1B). The number of incident PD patients decreased significantly compared to the past and has remained around 800 in recent years, a $4 \%$ incidence. Although the number of KT recipients gradually increased, the proportion was maintained at $12 \%$ to $14 \%$.

Consistent with the trend of ESRD incidence, the prevalence of RRT was increasing (Fig. 1C), finally surpassing 100,000 patients at the end of 2019. Among a total of 108,873 patients receiving RRT, 81,760 (75.1\%) were receiving HD, 5,960 (5.5\%) PD, and 21,153 (19.4\%) KT. The prevalence of ESRD has doubled since 2010, with Korea ranking sixth in the world with an incidence of 1,816 per million population (Fig. 1D, data extracted from the United States Renal Data System Annual Report [1]).

Underlying causes of ESRD and status of the elderly dialysis population

Over the past three decades, the three leading causes of ESRD in Korea were diabetes mellitus (DM), hypertension (HTN), and chronic glomerulonephritis (GN). The proportion of DM patients increased from 1992 (19.5\%) to 2012 (50.6\%). Currently, DM (48.4\%) is the most common cause of ESRD in Korea (Fig. 2A). Although this increase has plateaued since 2012, it was still higher than that in Japan or the United States $[1,6]$. The percentage of ESRD patients who had a kidney disease of unknown origin still exceeds $10 \%$ in recent years. In addition, the percentages of primary diseases such as polycystic kidney disease and miscellaneous diseases have remained constant.
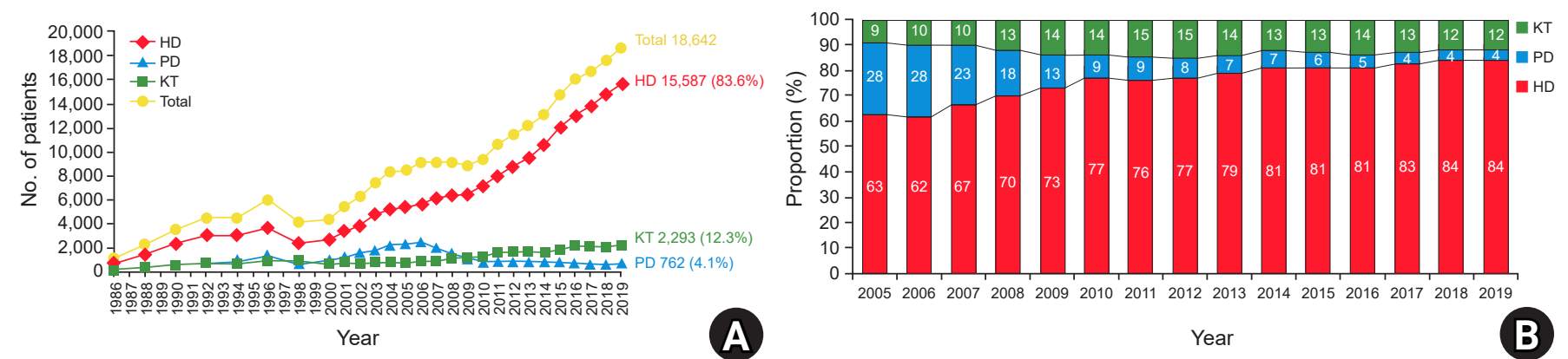

Year

B
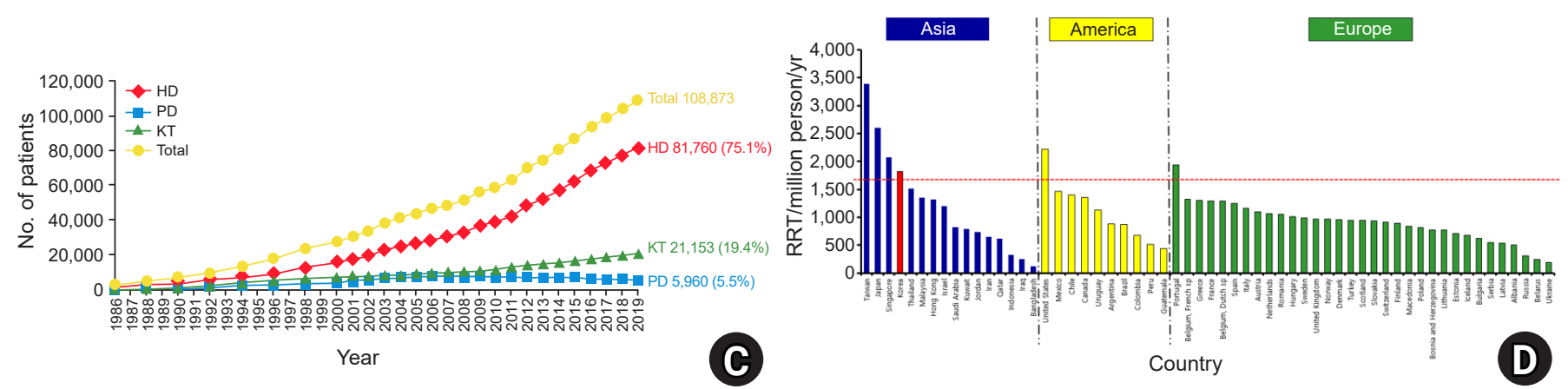

Figure 1. Incidence and prevalence of renal replacement therapy in Korea. (A) Annual incidence of RRT in Korea. (B) Proportion of renal replacement modalities (annual incidence from 1986 to 2019). (C) Annual prevalence of RRT in Korea. (D) The current status of RRT (per million population) in Korea through internal comparisons.

HD, hemodialysis; KT, kidney transplantation; PD, peritoneal dialysis; RRT, renal replacement therapy. 

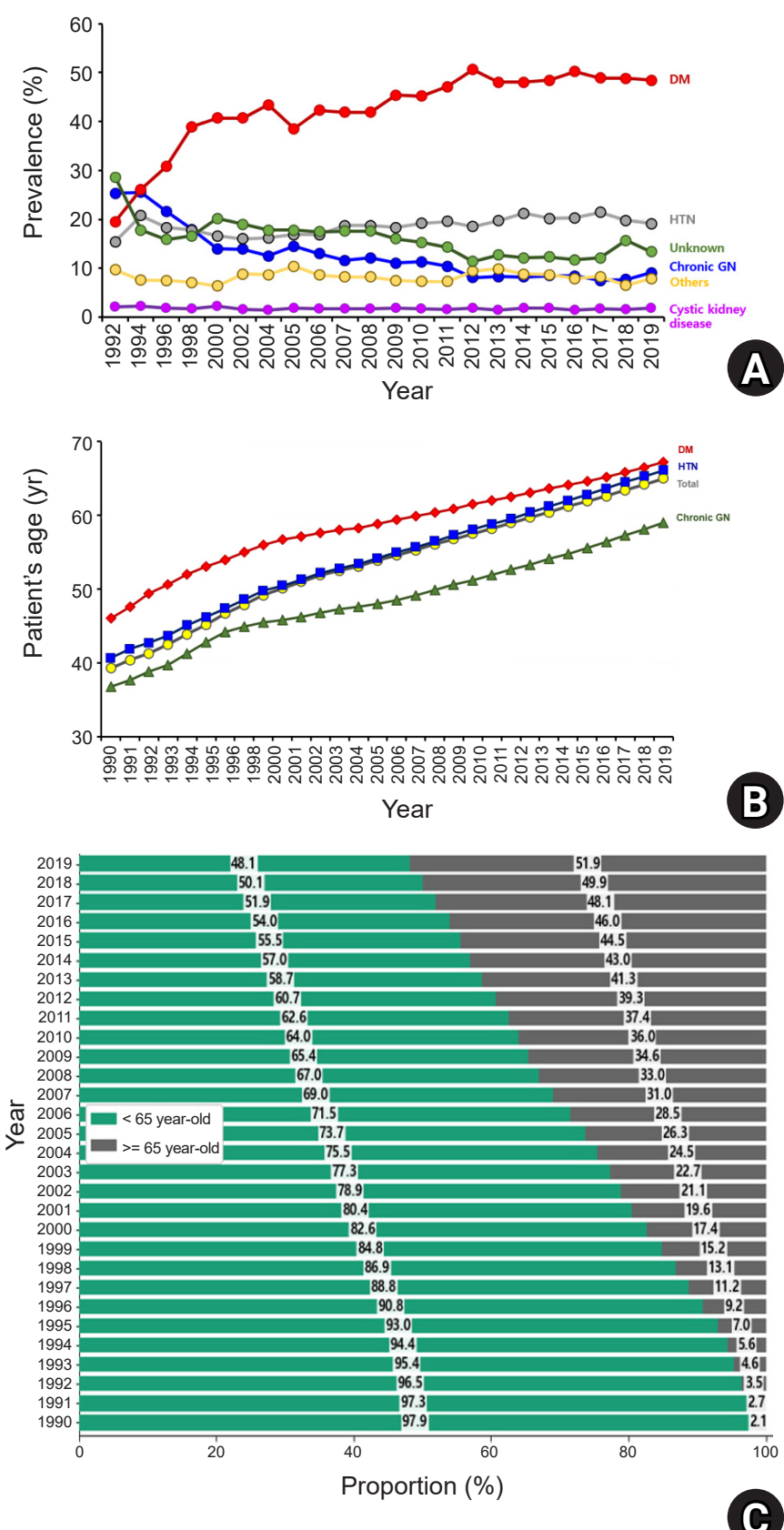

Figure 2. Prevalent percentages based on underlying cause of end-stage renal disease and age distribution in Korea. (A) Underlying causes of ESRD. (B) Mean ages of dialysis patients according to primary renal disease. (C) The proportion of dialysis patients over 65 years old.

DM, diabetes mellitus; ESRD, end-stage renal disease; GN, glomerulonephritis; HTN, hypertension.

Currently, there is a trend toward an increasing elderly population in Korea. In 2017, Korea achieved an aged society, with $14.02 \%$ of the population older than 65 years, and the country is rapidly approaching a super-aged society. Statistics Korea forecast that Korea will be a super-aged society in 2025 [7]. Regarding this aging trend, the dialysis population was consistent with the general population. The mean age of the overall dialysis population was 65.0 years in 2019 (Fig. 2B). The mean ages of patients who required dialysis due to DM and HTN were 67.2 and 66.1 years, respectively, higher than the 59.0 years reported by chronic GN. Finally, the proportion of dialysis patients over 65 years of age in Korea exceeded 50\% in 2019 (51.9\%) (Fig. 2C). The proportion of dialysis patients aged 65 years and older increased from approximately $25 \%$ in 2005 to more than $50 \%$ in 14 years. The proportion of patients on dialysis for more than 10 years was $9.0 \%$ for $\mathrm{HD}$ and $2.9 \%$ for PD 20 years ago, increasing to $19.3 \%$ for HD and $12.5 \%$ for PD 10 years later. At present, about $40 \%$ of HD patients and $50 \%$ of PD patients have been on dialysis for more than 10 years.

\section{Distribution of dialysis machines}

The increased total number of ESRD patients was mainly related to the rapid increase in HD patients, which led to an increase in the number of HD centers in Korea. At the end of 2019, the number of HD centers exceeded 1,000, and the number of HD machines exceeded 30,000 (Fig. 3). The number of HD machines per HD center has been maintained at 27 to 29 for about 10 years.

\section{All-cause mortality and survival rates by treatment modality}

A total of 149,947 eligible incident and prevalent dialysis patients between 1 January 2001 and 31 December 2019 was analyzed. During the median follow-up period of 34.8 months, 30,852 patients undergoing HD (23.8\%) and 8,429 patients undergoing PD (33.0\%) died, and mortality rates were 50.7 for HD and 89.3 for PD per 1,000 patient-years, respectively. In 2001, the unadjusted mortality rate for overall dialysis patients was 122.5 per 1,000 patient-years. By dialysis modality, mortality rates were 119.3 for HD patients and 121.6 for PD patients per 1,000 patient-years. In 2018, the unadjusted mortality rate for dialysis patients was 45.18 per 1,000 patient-years, and mortality rates by dialysis modality were 45.10 for HD patients and 51.33 for PD patients per 1,000 patient-years (Fig. 4A). The 5-year 


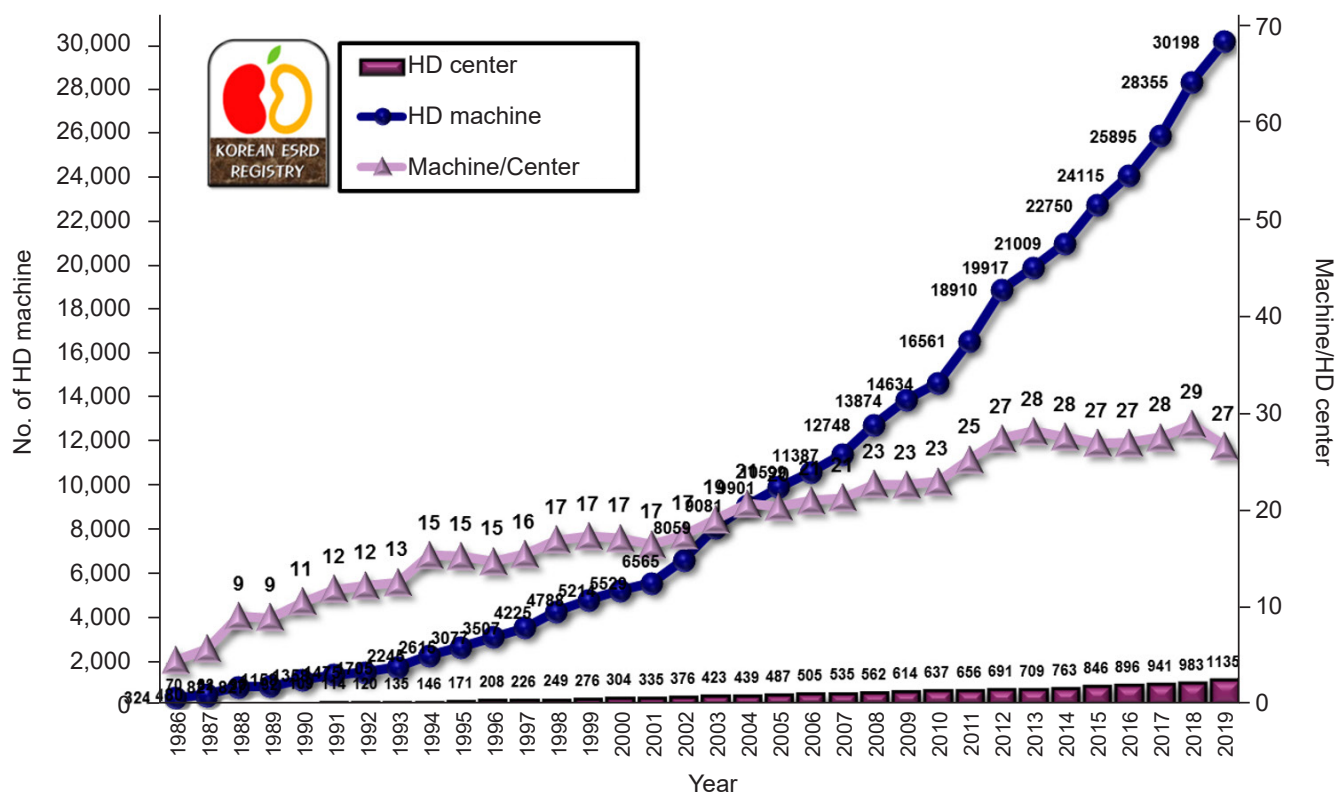

Figure 3. Numbers of hemodialysis (HD) centers and HD machines in Korea.

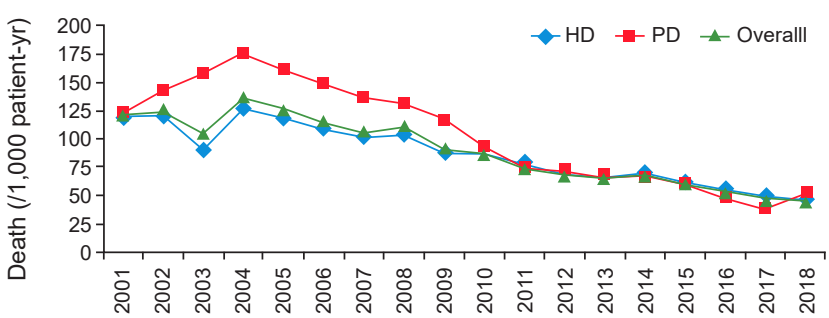

Year

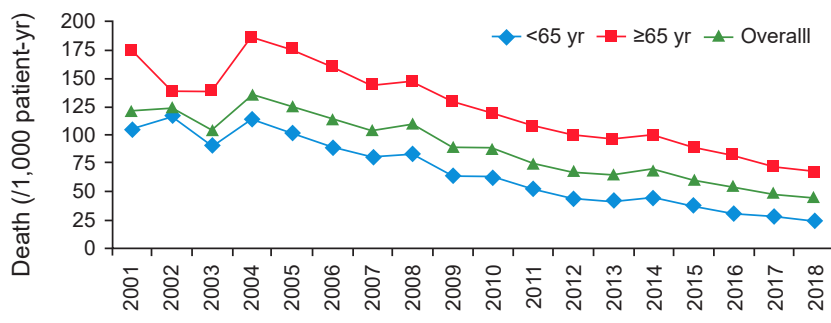

Year

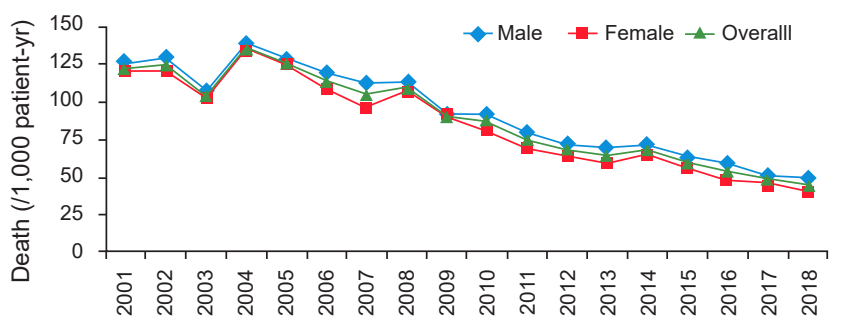

(A)

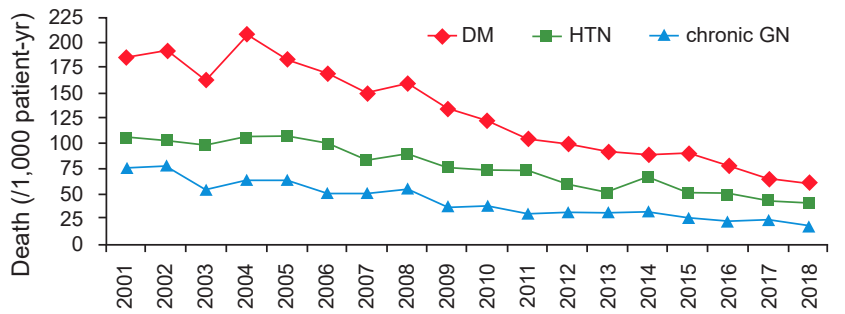

Year

Figure 4. Unadjusted all-cause mortality for all-dialysis patients, 2001 to 2018. (A) Treatment modality, (B) sex, (C) age, and (D) primary renal diseases.

DM, diabetes mellitus; GN, glomerulonephritis; HD, hemodialysis; HTN, hypertension; PD, peritoneal dialysis.

patient survival rate of dialysis patients after the onset of ESRD in 2001 was $69.0 \%$ in HD patients and $52.4 \%$ in PD patients. The 5-year patient survival rate after the onset of ESRD gradually increased from 2001 to 2013 in both HD and PD. Since 2013, the patient survival rate after the onset of ESRD in PD has been similar to that in HD. In 2016, the 3 month- and 1-, 2-, and 3-year patient survival rates after the onset of ESRD were $99.3 \%, 96.7 \%, 91.6 \%$, and $86.4 \%$ in HD, respectively, and $99.2 \%, 98.0 \%, 94.5 \%$, and $88.1 \%$ in PD. In 2019 , the 3 month- and 1-year patient survival rates after the 
onset of ESRD were $99.4 \%$ and $97.2 \%$ in HD, respectively, and $99.5 \%$ and $95.6 \%$ in PD (Table 1 ).

\section{Comparison of mortality rates by sex, age, and primary renal disease}

There was no significant difference between males and females in mortality rates for overall dialysis patients, and the change in mortality rates from 2001 to 2018 was similar between males and females. In 2001 and 2018, the unadjusted mortality rates per 1,000 patient-years were 125.0 and 48.6 for males, respectively, and 119.3 and 40.7 for females (Fig. 4B). The mortality rates of dialysis patients over 65 years of age were significantly higher than those of dialysis patients younger than 65 years from 2001 to 2018 (Fig. 4C). In 2001 and 2018, the unadjusted mortality rates per 1,000 patient-years were 174.7 and 67.3 for older patients, respectively, and 104.4 and 24.4 for younger patients. The mortality rates gradually decreased in both older and younger patients from 2001 to 2018. The mortality rates for diabetic patients were significantly higher than those of nondiabetic patients from 2001 to 2018. In 2001 and 2018, the unadjusted mortality rates per 1,000 patient-years were 184.9 and 59.4 for diabetic patients, respectively, and 96.3 and 35.5 for nondiabetic patients. The mortality rates of DM as a cause of ESRD were significantly higher than those

Table 1. Cumulative unadjusted survival rates (\%) by treatment modality and incident cohort year (year of end-stage renal disease onset)

\begin{tabular}{lccccc}
\hline Year & $3 \mathrm{Mo}$ & $12 \mathrm{Mo}$ & $24 \mathrm{Mo}$ & $36 \mathrm{Mo}$ & $60 \mathrm{Mo}$ \\
\hline $\begin{array}{l}\text { Hemodialysis } \\
2001\end{array}$ & 98.7 & 93.9 & 86.4 & 80.1 & 69.0 \\
2004 & 98.4 & 94.8 & 88.6 & 82.2 & 71.1 \\
2007 & 99.0 & 95.8 & 90.4 & 84.7 & 73.4 \\
2010 & 99.0 & 95.8 & 91.5 & 87.0 & 77.5 \\
2013 & 99.4 & 96.4 & 91.9 & 87.1 & 77.0 \\
2016 & 99.3 & 96.7 & 91.6 & 86.4 & - \\
2019 & 99.4 & 97.2 & - & - & - \\
Peritoneal dialysis & & & & & \\
2001 & 97.6 & 92.2 & 82.7 & 73.0 & 52.4 \\
2004 & 98.1 & 91.9 & 82.6 & 72.7 & 58.2 \\
2007 & 99.0 & 95.4 & 86.5 & 77.5 & 63.7 \\
2010 & 98.6 & 96.0 & 91.1 & 83.7 & 70.9 \\
2013 & 99.2 & 96.6 & 90.9 & 84.5 & 71.5 \\
2016 & 99.2 & 98.0 & 94.5 & 88.1 & - \\
2019 & 99.5 & 95.6 & - & - & - \\
\hline
\end{tabular}

of HTN and chronic GN from 2001 to 2018 (Fig. 4D). In 2001 and 2018, the unadjusted mortality rates per 1,000 patientyears were 184.9 and 59.4 for DM, 104.8 and 39.6 for HTN, and 76.5 and 19.0 for chronic GN, respectively.

\section{Cause-specific mortality rates}

The largest category of known cause-specific mortality for dialysis patients was death due to cardiac disease (35.8\%) in 2019. Non-uremic cardiac arrest, uremic cardiac arrest, and coronary artery disease were included in the category of cardiac disease. Non-uremic cardiac arrest, uremic cardiac arrest, and coronary artery disease comprised $15.3 \%$, $12.9 \%$, and $7.6 \%$ of known causes of death among dialysis patients, respectively. Infection-related disease (22.9\%) was the second most prevalent category of known causespecific mortality for dialysis patients. Sepsis, pneumonia, tuberculosis, and peritonitis comprised $11.2 \%, 8.2 \%, 0.6 \%$, and $0.1 \%$ of known causes of death among dialysis patients, respectively. Vascular disease $(11.2 \%)$ was the third most common cause of mortality for dialysis patients. Vascular disease consisted of cerebrovascular accident, pulmonary embolism, gastrointestinal hemorrhage, gastrointestinal embolism, and other vascular diseases. Treatment refusal, suicide, and treatment withdrawal for other reasons were included in social causes of death, and malnutrition, malignancy, accident, and uncertain cause were included in miscellaneous causes of death. Cerebrovascular accidents comprised $6.5 \%$ of known causes of death, malignancy accounted for $5.0 \%$, liver failure for $2.3 \%$, suicide for $0.8 \%$, and treatment refusal for $0.3 \%$. The cause of death information was missing or unknown for $19.0 \%$ of dialysis patients (Fig. 5). The trends of cause-specific death from 2001 to 2019 are presented in Fig. 6. The proportion of cardiac disease increased slightly and the proportion of vascular disease decreased. Consequently, the proportion of cardiovascular disease-related death has changed little from 2001 to 2019 among the known causes of death in dialysis patients. The proportion of infection-related death has slightly increased over 20 years (Fig. 6).

\section{Discussion}

In the 2019 report of the KORDS, major findings included rapid growth in the overall number of patients undergoing 

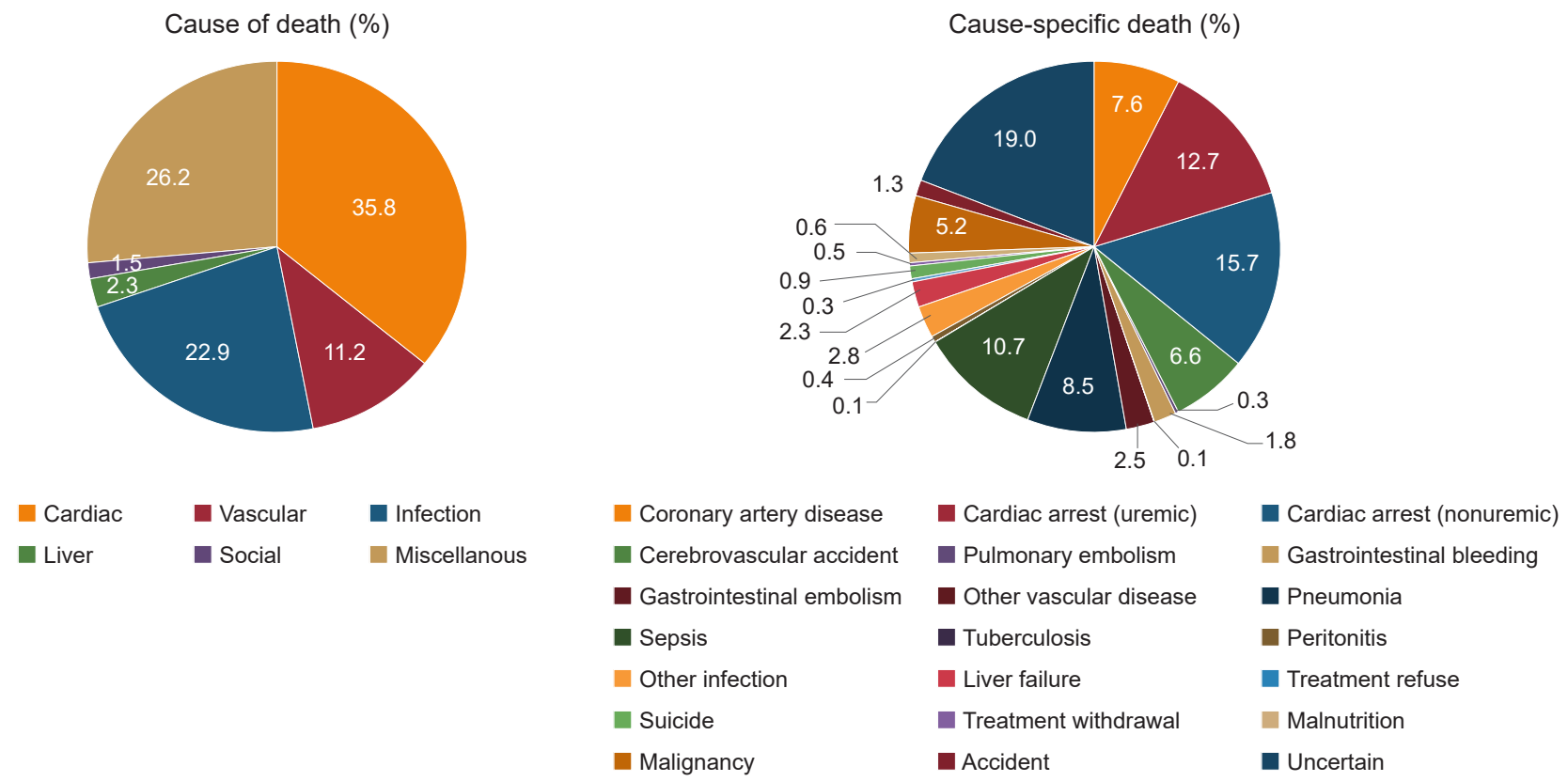

Figure 5. Unadjusted relative percentages for cause of death of dialysis patients in 2019. (A) Major causes of death and (B) detailed cause-specific death.

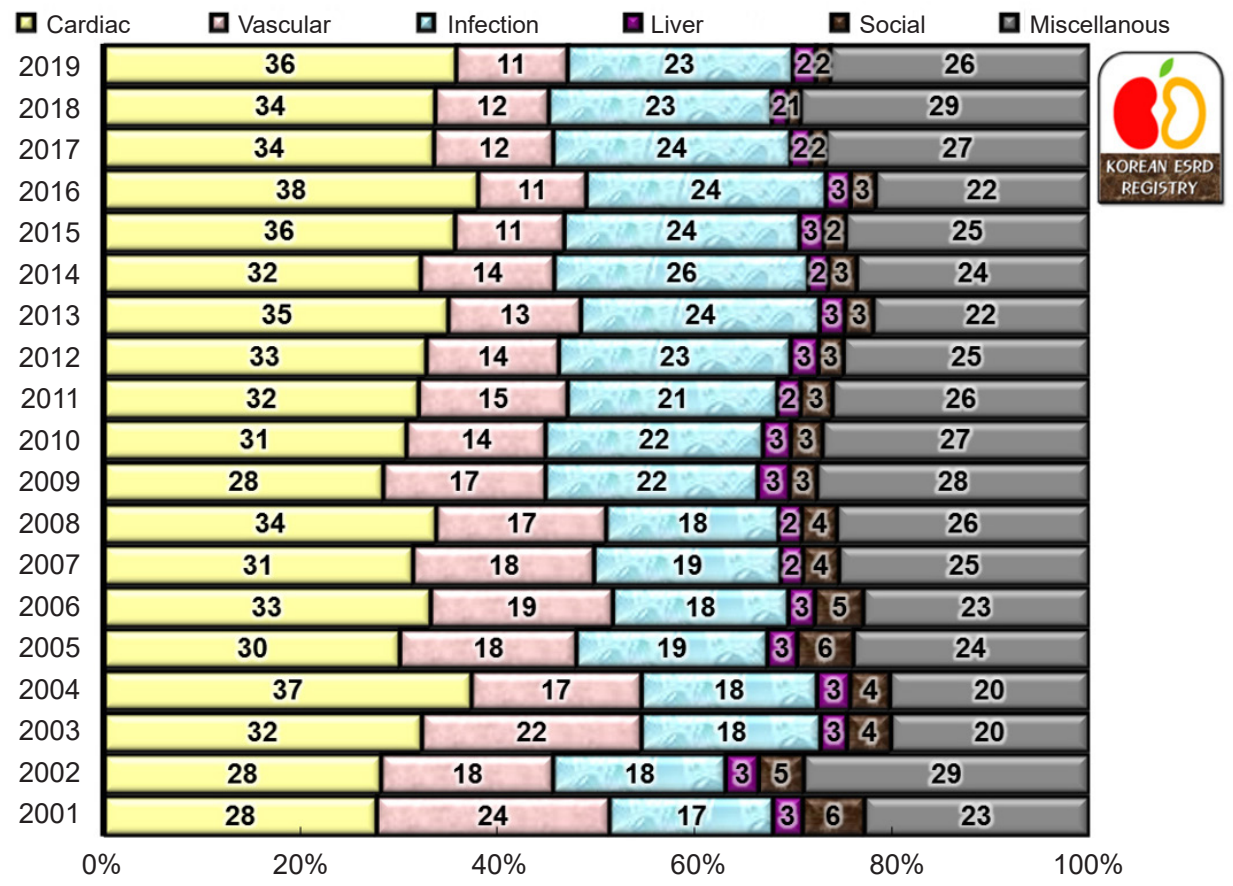

Figure 6. Trends for proportions of causes of death during the incident year, 2001 to 2019.

dialysis, the proportion of elderly patients, and the overall number of patients undergoing long-term dialysis. The proportion of diabetic ESRD patients was maintained at around $50 \%$, and a consistent decrease in the proportion of PD patients was observed in recent years. Among ESRD patients, the HD proportion in the study period increased to $84 \%$, and the PD proportion decreased to $4 \%$. Over the past two decades, the mortality of dialysis patients gradually 
decreased, but the mortality of diabetic dialysis patients was higher than that of dialysis patients with HTN or chronic GN. Recently, the patient survival rates in HD were similar to those in PD. Taken together, the survival improvement of dialysis patients and the increase in the proportion of longterm dialysis patients might contribute to the increase in the mean age of dialysis patients.

The incidence and prevalence of ESRD patients in Korea have been increasing over the past three decades, and the prevalence has doubled in the last 10 years. Although the prevalence of predialysis chronic kidney disease (CKD) stage 3 to 5 patients has remained constant during the same period [8], the number of ESRD patients requiring RRT showed a recent rapid increase. As a result, Korea is now ranked sixth in the world for prevalence of ESRD [9].

Although the data from KORDS in 2013 reported that the prevalence of ESRD patients did not appear to reach the inflection point [10], the value has been continually increasing and has now exceeded those of Japan and the United States. At present, the trend resembles that of Taiwan, where the overall number of ESRD patients increased at the greatest rate among global countries [9]. The data from KORDS showed that most ESRD patients underwent HD. In the future, the aging trend will contribute to a progressively higher modality rate of HD compared to PD and KT. Under such circumstances, the numbers of HD centers and dialysis machines are likely to increase.

To reduce the number of ESRD patients, it is necessary to improve adherence through the proper administration of prescribed agents and lifestyle modifications according to the causative disease and to reduce complications through nephrologist counseling from the early stage of CKD. In addition, the rate of ESRD cases of unknown origin remains high, which must be resolved to reduce ESRD in the long term. Fortunately, regular health screenings via school, workplace, and regional systems have been expanded in Korea [11]. Therefore, if a patient does not miss a regular health examination, data of unknown origin will gradually decrease through diagnosis of the causative disease.

Another interesting finding was that the reduction in mortality risk was greater for patients treated with PD than those treated with HD in the past two decades, and that the survival of patients treated with PD and HD is similar in Korea for recent years. Comparisons between the longterm survival rates of patients with $\mathrm{HD}$ and PD in the past two decades were first reported in 2019 using the KORDS. In Korea, a previous population-based, large-scale study using Korean Health Insurance Review and Assessment data suggested that the mortality rate was significantly higher in incident PD patients than in incident HD patients in the period from 2005 to 2008 [12]. On the other hand, PD was associated with better survival than HD in the early period of dialysis in a national prospective cohort study performed since 2009 [13]. The present data from KORDS showed higher overall mortality rates in PD patients than in HD patients in the 2000s. Although the reason why PD patients have shown recent improvement in outcomes is not clear, it may be attributed to increased reimbursement for PD in the national health insurance program, the development of PD education programs, and the use of biocompatible PD solutions. Recent nationwide cohort data also indicated that PD and in-center HD provide similar survival in Europe and the United States $[1,14]$. Because statistical analyses for outcomes in this registry data were conducted without adjustment for confounding variables, further research is needed to assess the exact trends of mortality rates by treatment modality in Korean ESRD patients.

Despite a substantially decreasing trend of mortality rates in the last 20 years, the absolute mortality risk remains high in dialysis patients due to cardiovascular events and infections. In the KORDS for cause-specific death, cardiovascular disease was the most common, accounting for $47.0 \%$ of deaths (cardiac disease, $35.8 \%$ and vascular disease, $11.2 \%$ ). Infection accounted for $22.9 \%$ of deaths and malignancy accounted for $5.0 \%$ of deaths in 2019 . Trends in cause of death over time remained unchanged for cardiovascular disease and exhibited a mild increase for infection from 2001 to 2019. Other nationwide registry data demonstrated a decrease in cardiovascular mortality in dialysis patients over time in Japan and Europe $[6,15]$. However, survival analyses of the KORDS have some limitations, including relatively low enrollment rates, a lack of information on patient survival, and misclassification of cause-specific death, due to data collection based on voluntary enrollment. Therefore, it is necessary to consider a plan to increase the enrollment rate of the KORDS.

In conclusion, the KSN ESRD registry showed substantial increases in ESRD incidence and prevalence, especially in elderly and diabetic groups. These data highlight the need to pay attention to the high mortality rates of elderly and 
diabetic dialysis patients in Korea. These data also indicate the need for evidence-based treatment approaches for elderly and diabetic patients to prevent the progression of kidney disease. Although recent survival rates were similar between HD and PD patients, the proportion of prevalent PD patients gradually decreased. Therefore, further research may be needed for the individualized risk prediction of mortality regarding the selection of dialysis modality in patients who will start RRT.

\section{Conflicts of interest}

All authors have no conflicts of interest to declare.

\section{Funding}

This work was supported by a National Research Foundation of Korea grant funded by the Korean government (MSIT) (2018R1C1B5045006).

\section{Acknowledgements}

The ESRD Registry Committee of the KSN thanks all of the medical doctors and nurses of the dialysis centers in Korea for participating in this registry.

\section{Authors' contributions}

Conceptualization: JP, YKK

Data curation: CYK

Formal analysis: SDH, SRC, HL

Investigation: HYJ, KK (Kyeongmin Kim), YEK

Methodology: SHK, THK

Visualization: HSK, CYY, KK (Kiwon Kim)

Writing-original draft: YAH, THB

Writing-review \& editing: JP, YKK

All authors read and approved the final manuscript.

\section{ORCID}

Yu Ah Hong, https://orcid.org/0000-0001-7856-4955 Tae Hyun Ban, https://orcid.org/0000-0002-2884-4948 Chae-Yeong Kang, https://orcid.org/0000-0001-8587-7765 Sun Deuk Hwang, https://orcid.org/0000-0003-0074-6972 Sun Ryoung Choi, https://orcid.org/0000-0002-9668-3349
Hajeong Lee, https://orcid.org/0000-0002-1873-1587

Hee-Yeon Jung, https://orcid.org/0000-0003-0232-7202

Kyeongmin Kim, https://orcid.org/0000-0002-5414-4339

Young Eun Kwon, https://orcid.org/0000-0002-0843-9857 Su

Su Hyun Kim, https://orcid.org/0000-0003-3382-528X

Tae Hee Kim, https://orcid.org/0000-0002-3001-234X

Ho-Seok Koo, https://orcid.org/0000-0001-7856-8083

Chang-Yun Yoon, https://orcid.org/0000-0001-8545-9344

Kiwon Kim, https://orcid.org/0000-0002-2885-0053

Jongha Park, https://orcid.org/0000-0002-1461-9483

Yong Kyun Kim, https://orcid.org/0000-0002-1871-3549

\section{References}

1. United States Renal Data System (USRDS). 2019 USRDS annual data report: epidemiology of kidney disease in the United States. Bethesda (MD): National Institutes of Health, National Institute of Diabetes and Digestive and Kidney Diseases, 2019 [cited 2020 Oct 21]. Available from: https://www.usrds.org/annual-datareport/.

2. Himmelfarb J, Vanholder R, Mehrotra R, Tonelli M. The current and future landscape of dialysis. Nat Rev Nephrol 2020;16:573585.

3. Jin DC, Han JS. Renal replacement therapy in Korea, 2012. Kidney Res Clin Pract 2014;33:9-18.

4. Jin DC. Dialysis registries in the world: Korean dialysis registry. Kidney Int Suppl 2015;5:8-11.

5. Jin DC, Yun SR, Lee SW, et al. Lessons from 30 years' data of Korean end-stage renal disease registry, 1985-2015. Kidney Res Clin Pract 2015;34:132-139.

6. The Japanese Society for Dialysis Therapy (JSDT). JSDT Renal Data Registry: annual dialysis data report for 2017 [Internet]. Tokyo: JSDT, 2017 [cited 2020 Oct 21]. Available from: https:// www.jsdt.or.jp/english/2426.html.

7. Statistics Korea. 2019 statistics on the aged [Internet]. Daejeon (Korea): Statistics Korea, 2019 [cited 2019 Sep 27]. Available from: http://kostat.go.kr/portal/eng/pressReleases/11/3/index. board.

8. Division of Chronic Disease Control; Korea Centers for Disease Control and Prevention. Korea Disease Control and Prevention Agency. Noncommunicable disease (NCD) statistics: trends in prevalence of chronic kidney disease (moderate to severe), 2008-2018. Public Health Wkly Rep 2020;13(11):618.

9. United States Renal Data System (USRDS). 2017 USRDS annual data report: epidemiology of kidney disease in the United States. 
Bethesda (MD): National Institutes of Health, National Institute of Diabetes and Digestive and Kidney Diseases, 2017 [cited 2020 Oct 21]. Available from: https://www.usrds.org/annual-datareport/previous-adrs/.

10. Jin DC. Major changes and improvements of dialysis therapy in Korea: review of end-stage renal disease registry. Korean J Intern Med 2015;30:17-22.

11. National Law Information Center; Ministry of Government Legistration. Enforcement Decree of the National Health Insurance Act [Internet]. Sejong (Korea): National Law Information Center, 2020 [cited 2020 Apr 7]. Available from: https://www.law.go.kr/법령/국민건강보험법.

12. Kim H, Kim KH, Park K, et al. A population-based approach indicates an overall higher patient mortality with peritoneal dialysis compared to hemodialysis in Korea. Kidney Int 2014;86:991-1000.

13. Choi JY, Jang HM, Park J, et al. Survival advantage of peritoneal dialysis relative to hemodialysis in the early period of incident dialysis patients: a nationwide prospective propensity-matched study in Korea. PLoS One 2013;8:e84257.

14. Kramer A, Boenink R, Noordzij M, et al. The ERA-EDTA registry annual report 2017: a summary. Clin Kidney J 2020;13:693-709.

15. Boenink R, Stel VS, Waldum-Grevbo BE, et al. Data from the ERA-EDTA Registry were examined for trends in excess mortality in European adults on kidney replacement therapy. Kidney Int 2020;98:999-1008. 